\title{
Bem-Estar Subjetivo, Violência e Sintomas de Depressão, Ansiedade e Estresse Em Adolescentes
}

\author{
Naiana Dapieve Patias ${ }^{1}$ \\ Universidade Federal do Rio Grande do Sul, Porto Alegre-RS, Brasil \\ Faculdade Meridional - IMED, Passo Fundo-RS, Brasil \\ Júlia Assumpção Heine, Débora Dalbosco Dell'Aglio \\ Universidade Federal do Rio Grande do Sul, Porto Alegre-RS, Brasil
}

\section{RESUMO}

Este estudo investigou relações entre exposição à violência direta (VD) e violência indireta (VI), Bem-Estar Subjetivo (BES) e sintomas de depressão, ansiedade e estresse em 426 adolescentes de 12 a 18 anos $(M=14,91 ; D P=1,66)$. Foram utilizados os instrumentos Triagem da Exposição de Crianças à Violência na Comunidade, Escala de Depressão, Ansiedade e Estresse para Adolescentes (EDAE-A), Escala de Afetos Positivos (AP) e Afetos Negativos (AN) e Escala Multidimensional de Satisfação de Vida para Adolescentes (EMSVA). A Regressão Linear Múltipla indicou um modelo em que ser exposto à VD e VI, ser do sexo feminino, ter maior nível de AN e menores níveis de SVA explicaram 47\% da variação nos escores da EDAE-A. Depressão e VD explicaram 39\% na variação nos escores do BES. A exposição à VD e VI foi associada a sintomas de ansiedade, depressão e estresse, demonstrando que se constitui num fator de risco ao desenvolvimento emocional.

Palavras-chave: violência; adolescência; depressão; ansiedade; maus-tratos.

\section{ABSTRACT - Subjective Well-Being, Violence and Symptoms of Depression, Anxiety and Stress in Adolescents} This study investigated the relationship between exposure to direct violence (DV) and indirect violence (IV), subjective well-being (SWB) and symptoms of depression, anxiety and stress in 426 adolescents aged 12 to 18 years $(M=14.91 ; S D=1.66)$. The instruments used were the Screening for Children's Exposure to Violence in the Community; Depression, Anxiety and Stress Scale for Adolescents (DASS-A); Positive Affects Scale (PA) and Negative Affects (NA) Scale; and the Multidimensional Scale of Life Satisfaction for Adolescents (MSLSA). Multiple Linear Regression indicated a model in which being exposed to DV and IV, being female, having a higher level of NA and lower levels of SVA, explained 47\% of the variation in DASS-A scores. Depression and DV explained 39\% of the variation in SWB scores. Exposure to DV and IV was associated with symptoms of anxiety, depression and stress, demonstrating that it constitutes a risk factor for emotional development.

Keywords: violence; adolescence; depression; anxiety; mistreatment.

\section{RESUMEN - Bienestar Subjetivo, Violencia y Síntomas de Depresión, Ansiedad y Estrés en los Adolescentes}

Este estudio investigó las relaciones entre exposición a violencia directa (VD), violencia indirecta (VI), Bienestar Subjetivo (BES) y síntomas de depresión, ansiedad y estrés en 426 adolescentes de 12 a 18 años $(M=14,91 ; D P=1,66)$. Los instrumentos utilizados fueron Proyección de Exposición de Niños a Violencia en la Comunidad; Escala de Depresión, Ansiedad y Estrés para adolescentes (EDAE-A); Escala de Afectos Positivos (AP) y Afectos Negativos (AN) y Escala Multidimensional de Satisfacción de Vida para adolescentes (EMSVA). La regresión lineal múltiple indicó un modelo, en que estar expuesto a VD y VI, ser de sexo femenino, tener mayor nivel de AN y niveles menores de SVA explica el 47\% de variación en los puntajes de EDAE-A. Depresión y VD explicaron el 39\% de variación en los puntajes del BES. La exposición a VD y VI se ha asociado con síntomas de ansiedad, depresión y estrés, demostrando que se constituye en un factor de riesgo para el desarrollo emocional.

Palabras clave: violencia; adolescencia; depresión; ansiedad; maltrato.

A adolescência é um período de vida em que ocorrem muitas mudanças biológicas, psicológicas e sociais, que podem levar a uma maior vulnerabilidade e exposição à violência (Franco \& Rodrigues, 2014). Aspectos individuais, familiares, comunitários, históricos, sociais e culturais apresentam-se como fatores que exercem grande influência na forma de vivenciar a adolescência. Assim, a adolescência deve ser considerada uma etapa peculiar do desenvolvimento, em que riscos também estão presentes nos seus contextos de inserção (Pratta \& Santos, 2007; Steinberg \& Lerner, 2004).

Adolescentes de diversos níveis socioeconômicos e culturais têm sido expostos de forma direta (ser vítima) e indireta (testemunhar ou ouvir falar) às mais variadas formas de violência. Essas violências ocorrem tanto na família (violência intrafamiliar) quanto fora dela (violência 
extrafamiliar) (Maranhão, Coêlho, Lopes, Colaço, \& Santos, 2014). Além disso, adolescentes mais velhos e do sexo masculino são o público mais vulnerável à exposição à violência quando comparados com adolescentes mais novos e meninas (Waiselfisz, 2014).

A exposição à violência, tanto de forma direta quanto indireta, tem sido associada a transtornos mentais (Benetti, Schwartz, Soares, Macarena, \& Patussi, 2014), que podem ser internalizantes (por exemplo, quando há sintomas de ansiedade, depressão e somatização); ou externalizantes, quando são observados sintomas impulsivos, de agressão, conduta disruptiva e abuso de substâncias (American Psychiatric Association, 2014). No entanto, os estudos sobre exposição à violência e problemas emocionais ou comportamentais tratam, principalmente, das consequências em termos externalizantes, predominantemente, no sexo masculino (Fowler, Tompsett, Braciszewski, Jacques-Tiura, \& Baltes, 2009; Mrug \& Windle, 2010). Embora menos clara, há associação entre violência e sintomas de internalização, como, por exemplo, a depressão, a qual evidencia-se mais relacionada ao sexo feminino (Mrug \& Windle, 2010; Margolin, Vickerman, Oliver, \& Gordis, 2010).

Dessa forma, a violência tem sido considerada um importante fator de risco para um desenvolvimento não saudável. No entanto, nem sempre há um desfecho negativo em termos de desenvolvimento. Assim, considera-se que a forma como a violência é experimentada e significada pelo sujeito pode contribuir para reações negativas ou a criação de formas de enfrentamento (Maranhão et al., 2014). De fato, apesar da maior parte dos estudos ressaltar o aumento de sintomas de externalização, em menor número, as pesquisas que investigam internalização identificam que nem todos adolescentes expostos à violência desenvolvem tais comportamentos (Maranhão et al., 2014; Fowler et al., 2009; Barry, Rabkin, Olezeski, Rivers, \& Gordis, 2015; Margolin \& Gordis, 2000).

Nesse processo, podem estar presentes fatores de proteção que moderam ou mediam os efeitos da exposição à violência. Por exemplo, em um estudo brasileiro realizado com adolescentes vítimas de violência doméstica, foi verificado que o apoio social dos amigos e da comunidade, seja de forma material, informativa ou afetiva, contribuiu para minimizar os efeitos da exposição à violência (Carlos, Ferriani, Esteves, Silva, \& Scatena, 2014). Outro estudo nacional sugere que, além de aspectos ambientais, características individuais, como a personalidade pode influenciar na resposta a estressores ambientais (Woyciekoski, Natividade, \& Hutz, 2014).

Assim, nem sempre os adolescentes desenvolvem algum tipo de reação negativa face à exposição à violência. Por exemplo, estudo de meta-análise (Fowler et al., 2009) indicou que a exposição à violência esteve mais associada à Transtorno de Estresse Pós-traumático (TEPT) e sintomas externalizantes do que a sintomas internalizantes, embora o TEPT seja considerado categoria desses últimos. Uma possível explicação dos autores para o menor efeito da exposição à violência nos sintomas de internalização refere-se à capacidade de adaptação de crianças e adolescentes frente a essas situações. Segundo Fowler et al. (2009), as vítimas podem sofrer dessensibilização e apresentar comportamentos desadaptativos frente ao evento, como deixar de reagir ou naturalizar a violência. A dessensibilização pode levar os adolescentes a não exibirem medo, tristeza ou evitação, mas pode levá-los a reagir de maneira agressiva.

Outro estudo, realizado com adolescentes afroamericanos expostos à violência na comunidade, indicou que a violência pode ser normativa e não levar ao mau ajustamento, já que os participantes demonstraram capacidade de adaptação às situações de violência crônica em que vivem (Carey \& Richards, 2014). Para os autores, viver em áreas com alta criminalidade pode dessensibilizar os adolescentes, o que, de certa forma, pode protegê-los do estresse emocional e dos efeitos negativos da exposição. Além disso, estudos afirmam que os adolescentes expostos cronicamente à violência podem desenvolver estratégias para enfrentar essas situações (Carey \& Richards, 2014; Maranhão et al., 2014).

A exposição à violência pode impactar de forma diferente cada adolescente, dependendo de vários aspectos, como tipo de exposição (direta ou indireta), contexto (intra ou extrafamiliar), tempo (duração da exposição), frequência, idade, sexo, entre outros fatores (Margolin \& Gordis, 2000). A identificação de aspectos saudáveis ou de variáveis protetivas ao desenvolvimento também deve fazer parte da prática e da pesquisa com adolescentes já que nem todos os adolescentes expostos à violência desenvolvem subsequentes sintomas emocionais ou comportamentais (Barry et al., 2015). Analisar quais são os fatores de proteção que mitigam os efeitos da violência é um aspecto essencial que deve fazer parte das pesquisas para aplicação e intervenção a fim de promover competências, maximizando potenciais e minimizando riscos da exposição à violência.

Uma das características protetivas ao desenvolvimento e que tem sido pouco estudada em adolescentes (Camargo, Abaid, \& Giacomoni, 2011), principalmente em situações de maior vulnerabilidade, é o bem-estar subjetivo (Long, Huebner, Wedell, \& Hills, 2012; Poletto \& Koller, 2011; Seligman \& Csikszentmihalyi, 2000). Este é entendido por diversos autores como um fenômeno amplo que inclui dois conceitos específicos: julgamentos globais de satisfação com a vida, ou com domínios específicos dela (avaliação cognitiva em domínios específicos da vida como trabalho, casamento, etc.), e experiências emocionais positivas e negativas (análise emocional e pessoal sobre a frequência com que experimentam emoções positivas e negativas) (Diener, 2000).

O bem-estar subjetivo é ainda compreendido como um fator protetivo que pode mitigar os riscos ao longo do desenvolvimento e ser um fator que promove e mantém 
bons níveis de saúde mental (Park, 2004). Para alguns autores (Woyciekoski et al., 2014), pessoas satisfeitas com suas vidas tendem a perceber eventos, mesmo os estressores, de forma mais positiva. Assim, adolescentes expostos à violência podem ou não apresentar sintomas decorrentes da exposição e, além disso, apresentar bem- estar. Por outro lado, podem não apresentar sintomas de depressão, ansiedade e estresse e, mesmo assim, não apresentar bem-estar. Ou seja, o bem-estar subjetivo depende da avaliação do próprio sujeito de forma que as circunstâncias subjetivas são mais importantes do que as objetivas (Franco \& Rodrigues, 2014). Estudos brasileiros, sob essa perspectiva, são realizados, principalmente, com adultos (Mendonça, Ferreira, Caetano, \& Torres, 2014; Scorsolini-Comin \& Santos, 2011) e idosos (Oliveira, Queiroz, \& Costa, 2012).

No entanto, alguns estudos brasileiros sobre bem-estar subjetivo com crianças e adolescentes são encontrados. Um estudo investigou o bem-estar subjetivo de 297 crianças e adolescentes com idades entre sete e 16 anos que viviam em instituições de acolhimento ou com suas famílias. A fim de avaliar o bem-estar subjetivo, as autoras utilizaram as escalas de afeto positivo e negativo e a escala multidimensional de satisfação de vida. Análises descritivas, ANOVA e teste $\mathrm{t}$ para comparar os construtos por grupos indicaram que, em termos de satisfação de vida (SV) e afetos positivos (AP), os grupos não diferiram. No entanto, o grupo que vivia em instituições de acolhimento apresentou maior média nos afetos negativos (AN), assim como as meninas em relação aos meninos (Poletto \& Koller, 2011). Segundo as autoras, uma hipótese importante para o fato de não haver diferenças entre os grupos na SV e nos AP refere-se ao contato com os irmãos (que também viviam nas instituições) e com parentes próximos no grupo que vive nas instituições de acolhimento. As mesmas autoras referem que a vivência de inúmeros eventos estressores pode aumentar os níveis de AN nas crianças e adolescentes que vivem em instituições.

Outro estudo nacional, realizado com 425 adolescentes com idades entre 14 e 19 anos estudantes de escolas públicas e privadas de uma cidade do interior do Rio Grande do Sul, verificou que as meninas experenciaram mais afetos negativos e menos afetos positivos do que os meninos. O resultado do estudo não encontrou diferenças estatisticamente significativas nos afetos positivos e negativos por faixa etária (adolescentes mais novos e mais velhos) e tipo de escola (pública e privada) (Segabinazi et al., 2012).

Um estudo com adolescentes do sul do Brasil (Segabinazi, Giacomoni, Dias, Teixeira, \& Moraes, 2010) indicou uma tendência a menores escores na satisfação de vida em adolescentes de 17 a 19 anos, quando comparados aos mais novos. Em relação ao sexo, os meninos apresentaram maiores níveis de satisfação de vida, quando comparados às meninas, na maior parte dos domínios avaliados (família, não violência, self comparado e autoeficácia). No entanto, os autores revelam que há inconsistências na literatura sobre as diferenças, em relação à idade e sexo, na satisfação de vida.

A fim de contemplar aspectos pouco explorados na literatura nacional, este estudo teve como objetivo verificar as relações entre exposição à violência direta (ser vítima) e indireta (testemunhar ou ouvir falar sobre), bem-estar subjetivo e sintomas de depressão, ansiedade e estresse em adolescentes escolares de 12 a 18 anos.

\section{Método}

\section{Participantes}

Este trabalho faz parte de um projeto de pesquisa maior intitulado "Exposição à violência na Adolescência: Relações com bem-estar e saúde mental", que tem por objetivo geral investigar a relação entre exposição a diferentes tipos de violência, bem-estar e saúde mental em adolescentes. Participaram 426 adolescentes, com idades entre 12 e 18 anos $(M=14,91 ; D P=1,66), 264$ meninas (62\%), estudantes do $6^{\circ}$ ano do Ensino Fundamental ao $3^{\circ}$ ano do Ensino Médio, de cinco escolas públicas de diferentes regiões da cidade de Porto Alegre (RS, Brasil), sendo uma escola localizada no centro da cidade e outras afastadas dele. Todas as escolas atendiam crianças e adolescentes em situação de vulnerabilidade social e foram observados relatos de professores e funcionários sobre situações de violência ocorridas dentro e fora da escola. $\mathrm{O}$ número de participantes da amostra foi obtido por meio do cálculo amostral, a partir do número total de alunos de Ensino Fundamental e Médio matriculados em escolas públicas de Porto Alegre, com uma margem de erro estabelecida de 5\% (Barbetta, 2001).

\section{Instrumentos}

Foram utilizados os seguintes instrumentos:

Ficha de dados sociodemográficos: teve como objetivo investigar dados, como idade, sexo, série escolar, configuração familiar, nível de escolaridade dos pais, repetência escolar, entre outros.

Escala Multidimensional de Satisfação de Vida para Adolescentes (EMSVA) (Segabinazi et al., 2010): composta por 52 itens que avaliam sete dimensões ou domínios, estabelecidos por meio de análise dos componentes principais, que interferem na avaliação cognitiva da satisfação de vida de adolescentes: família (ex.: Minha família se dá bem.), self (ex.: Eu sou uma pessoa bem humorada.), escola (ex.: Eu me sinto bem na minha escola.), self comparado (ex.: Meus amigos podem fazer mais coisas do que eu.), não violência (ex.: Mantenho a calma.), autoeficácia (ex.: Sou capaz de realizar muitas coisas.) e amizade (ex.: Gosto de conversar com meus amigos.). Os adolescentes devem indicar, em cada item, o número que melhor representa o quanto concorda com o que a frase diz sobre ele, em uma escala likert de cinco pontos, 
variando de 1 (nem um pouco) a 5 (muitíssimo). No estudo de desenvolvimento e validação preliminar da escala, realizado com 425 adolescentes de 14 a 19 anos, foi encontrada uma adequada consistência interna da escala $(\alpha=0,93)$ e em cada subitem, que apresentaram fidedignidade de 0,70 a 0,91 .

Escala Afetos Positivos e Negativos para Adolescentes (EAPNA) (Segabinazi et al., 2012): constitui-se de 28 adjetivos descritores de estados afetivos subjetivos, sendo alguns positivos (ex.: alegre, amoroso, disposto e feliz) e outros negativos (ex.: chateado, culpado, deprimido e impaciente). $\mathrm{O}$ adolescente deve responder como está se sentido ultimamente, em relação a cada adjetivo, com opções de resposta em formato de escala likert de cinco pontos, variando de "nem um pouco", "um pouco", "mais ou menos", "bastante" a "muitíssimo". O estudo de adaptação, normatização e validação, realizado com 425 adolescentes de 14 a 19 anos $(M=16,07 ; D P=1,12)$, verificou uma solução de dois fatores (AP e AN), a partir da análise dos componentes principais. A escala apresentou índices de consistência interna avaliados por meio do alfa de Cronbach de $\alpha=0,88$ para AP e para AN.

Índice Bem-Estar Subjetivo (IBES): O índice foi construído utilizando-se os escores padronizados da Escala Multidimensional de Satisfação de Vida para Adolescentes (EMSVA) somados aos escores padronizados das Escalas de Afetos Positivos e Negativos para Adolescentes (EAPNA). Cabe ressaltar que os itens de AN foram invertidos para o cálculo. Análise dos Componentes Principais foi realizada para a construção do compósito que deu origem ao Índice de Bem-Estar Subjetivo (IBES). Os componentes usados para criar o IBES são provenientes de modelo teórico (Diener, 2000; Segabinazi et al., 2010; Segabinazi et al., 2012), que indica a Satisfação com a Vida (SV), Afetos Positivos (AP) e Negativos (AN) como componentes do BES, sendo a $\mathrm{SV}$ o componente cognitivo e os AP e AN os componentes afetivos.

Escala de Depressão, Ansiedade e Estresse para adolescentes (EDAE-A) (Patias, Machado, Bandeira, \& Dell'Aglio, 2016): composta por três subescalas para avaliar sintomas de depressão, ansiedade e estresse. Cada subescala contém sete itens, respondidos em uma escala tipo likert de quatro pontos, onde os extremos são "Não aconteceu comigo nessa semana" (0) a "Aconteceu comigo na maior parte do tempo da semana" (3). O estudo de adaptação e validação foi realizado com 426 adolescentes de escolas públicas com idades entre 12 a 18 anos $(M=14,91$; $D P=1,66)$. Por meio de análise fatorial confirmatória, verificou-se uma solução de três fatores (depressão, ansiedade e estresse). Neste estudo, foram observadas medidas de fidedignidade de $\alpha=0,86$ para a subescala estresse; $\alpha=0,83$ para subescala ansiedade e $\alpha=0,90$ para subescala depressão. A escala total EDAE-A obteve índice de $\alpha=0,90$.

Triagem de Exposição de Crianças à Violência na Comunidade (Richters \& Martinez, 1993; adaptado no Brasil por Zavaschi, Benetti, Polanczyk, Solés, \& Sanchotene, 2002): Questionário desenvolvido pelo National Institute of Mental Health, nos Estados Unidos, para identificar crianças e adolescentes que foram envolvidos em situações de violência na comunidade (Richters \& Martinez, 1993). Consiste em 49 questões que abordam quatro tipos de violência - violência comunitária, familiar, sexual e exposição às drogas, das quais os adolescentes possam ter sido as próprias vítimas da violência (exposição direta ou violência direta), possam ter presenciado como testemunhas, ou possam ter ouvido falar sobre tais situações (exposição indireta ou violência indireta), e uma questão aberta para que situações não listadas sejam identificadas. As questões são respondidas por meio de escolha simples, devendo o adolescente marcar verdadeiro ou falso caso tenha ou não sido vítima da situação. Exemplo de situações de exposição direta à violência: Eu recebi tapas, socos ou apanhei de algum membro da minha família; Eu estava em casa quando alguém invadiu ou tentou invadir a minha casa/apartamento; Exemplos de afirmativas de exposição indireta à violência: Eu conheço uma pessoa que apanhou ou foi assaltada; Eu vi ou ouvi um revólver ser disparado enquanto eu estava em casa. No estudo de tradução e adaptação para o Brasil (Zavaschi et al., 2002), foi observada uma consistência interna de $\alpha=0,89$.

\section{Procedimentos}

A pesquisa da qual este estudo faz parte foi aprovada por Comitê de Ética em Psicologia (CAAE 22080914.1.0000.5334) e seguiu os aspectos éticos que garantem a integridade dos participantes, conforme previsto na Resolução $n^{\circ} 466$ (Brasil, 2013). As escolas públicas foram selecionadas por conveniência, em diferentes regiões da cidade de Porto Alegre, RS. Após apresentação do projeto de pesquisa e assinatura do Termo de Concordância de cada escola, turmas de Ensino Fundamental e Médio de cada escola foram convidadas a participar. Foi solicitado o Termo de Consentimento Livre e Esclarecido (TCLE) para os pais ou cuidadores e o Termo de Assentimento para os adolescentes que concordaram em participar. A aplicação dos instrumentos foi realizada em sala de aula, em grupo de aproximadamente 20 adolescentes. Os resultados parciais foram apresentados para as turmas, após o término da coleta de dados e posterior análise.

\section{Análise de Dados}

Os dados foram armazenados e analisados no programa estatístico SPSS, versão 22.0. Foram realizadas análises descritivas, observando-se médias, desvios-padrão, medianas, mínimo e máximo das variáveis investigadas. A fim de verificar a normalidade das variáveis investigadas (VD e VI, BES, depressão, ansiedade e estresse) foram considerados os valores de $p>0,05$ para o teste de Kolmogorov-Smirnov. A inexistência de 
multicolinearidade foi investigada admitindo-se valores VIP maiores que 5 e tolerância maiores que 0,2.

A análise de regressão linear múltipla, pelo método stepwise, investigou as variáveis associadas à escala EDAE-A e ao Índice de Bem-Estar Subjetivo (IBES) (construído por meio dos escores de SV, AP e AN). Além disso, foi calculado tamanho de efeito $r$ para cada variável independente.

Análises de correlação de Pearson indicaram baixa correlação entre a escala de Afetos Positivos e de Afetos Negativos $(r=-0,35 ; p<0,001)$. Dessa forma, foram avaliados como conceitos independentes, assim como em outros estudos nacionais (Polleto \& Koller, 2011). O índice foi construído utilizando-se os escores padronizados da Escala Multidimensional de Satisfação de Vida para Adolescentes (EMSVA) somados aos escores padronizados das Escalas de Afetos Positivos e Negativos para Adolescentes (EAPNA). Cabe ressaltar que os itens de AN foram invertidos para o cálculo.

\section{Resultados}

A Tabela 1 apresenta os dados descritivos (médias, medianas, mínimo e máximo dos escores) das variáveis investigadas.

Tabela 1

Dados Descritivos das Variáveis Investigadas

\begin{tabular}{|c|c|c|c|c|c|}
\hline Variável & $M$ & $D P$ & Md & Mínimo & Máximo \\
\hline Violência Direta & 1,64 & 1,77 & 1 & 0 & 8 \\
\hline Violência Indireta & 12,94 & 7,96 & 13 & 0 & 34 \\
\hline EDAE-A & 12,90 & 10,57 & 10 & 0 & 57 \\
\hline Subescala depressão & 4,27 & 4,43 & 3 & 0 & 21 \\
\hline Subescala ansiedade & 2,98 & 3,39 & 2 & 0 & 19 \\
\hline Subescala estresse & 5,65 & 4,54 & 5 & 0 & 20 \\
\hline IBES & 225,72 & 42,89 & 231 & 69 & 311 \\
\hline Afeto Positivo & 50,91 & 52 & 10,89 & 14 & 70 \\
\hline Afeto Negativo & 24,77 & 22 & 10,08 & 14 & 63 \\
\hline EMSVA (SV) & 199,60 & 201 & 28,12 & 100 & 256 \\
\hline
\end{tabular}

Nota. EDAE-A=Escala de Depressão, Ansiedade e Estresse versão Adolescentes; IBES=Índice de Bem-Estar Subjetivo; EMSVA=Escala Multidimensional de Satisfação de Vida (SV) para Adolescentes

Na Tabela 2, são apresentadas as correlações obtidas por meio do teste de Pearson entre as variáveis do estudo: idade, sexo, VD, VI, EDAE-A e as subescalas depressão, ansiedade e estresse, SV, AP, AN e IBES. A análise indicou que há correlações significativas entre a escala EDAE-A com quase a totalidade das variáveis, com exceção da idade. O mesmo foi verificado em relação ao IBES.

Tabela 2

Matriz de Correlações

\begin{tabular}{|c|c|c|c|c|c|c|}
\hline & Sexo & $\begin{array}{l}\text { Violência } \\
\text { Direta }\end{array}$ & $\begin{array}{l}\text { Violência } \\
\text { Indireta }\end{array}$ & EDAE-A & $\begin{array}{c}\text { Afeto } \\
\text { Positivo }\end{array}$ & $\begin{array}{c}\text { Afeto } \\
\text { Negativo }\end{array}$ \\
\hline Idade & 0,02 & $0,29^{* *}$ & $0,31^{* *}$ & 0,03 & 0,06 & $-0,01$ \\
\hline Sexo & - & 0,07 & 0,05 & $-0,23^{* *}$ & 0,03 & $-0,16^{* *}$ \\
\hline VD & - & - & $0,59^{* *}$ & $0,38^{* *}$ & $-0,05$ & $0,28^{* *}$ \\
\hline VI & - & - & - & $0,36^{* *}$ & 0,03 & $0,27^{* *}$ \\
\hline EDAE-A & - & - & - & - & $-0,28^{* *}$ & $0,59^{* *}$ \\
\hline AP & - & - & - & - & - & $-0,35^{* *}$ \\
\hline AN & - & - & - & - & - & - \\
\hline SV & - & - & - & - & - & - \\
\hline IBES & - & - & - & - & - & - \\
\hline Depressão & - & - & - & - & - & - \\
\hline \multirow[t]{2}{*}{ Ansiedade } & - & - & - & - & - & - \\
\hline & $\begin{array}{c}\text { Satisfação } \\
\text { Vida }\end{array}$ & IBES & \multicolumn{2}{|c|}{ Depressão } & Ansiedade & Estresse \\
\hline Idade & $-0,02$ & 0,00 & \multicolumn{2}{|c|}{0,00} & 0,05 & 0,03 \\
\hline
\end{tabular}


Tabela 2 (continuação)

Matriz de Correlações

\begin{tabular}{lccccc}
\hline & $\begin{array}{c}\text { Satisfação } \\
\text { Vida }\end{array}$ & IBES & Depressão & Ansiedade & Estresse \\
\hline Sexo & $00,11^{*}$ & $0,11^{*}$ & $-0,19^{* *}$ & $-0,16^{* *}$ & $-0,22^{* *}$ \\
VD & $-00,24^{* *}$ & $-0,24^{* *}$ & $0,25^{* *}$ & $0,35^{* *}$ & $0,37^{* *}$ \\
VI & $-00,15^{* *}$ & $-0,15^{* *}$ & $0,21^{* *}$ & $0,35^{* *}$ & $0,36^{* *}$ \\
EDAE-A & $-00,47^{* *}$ & $-0,52^{* *}$ & $0,85^{* *}$ & $0,82^{* *}$ & $0,88^{* *}$ \\
AP & $0,72^{* *}$ & $0,81^{* *}$ & $-0,41^{* *}$ & $-0,13^{* *}$ & $-0,15^{* *}$ \\
AN & $-0,54^{* *}$ & $-0,67^{* *}$ & $0,64^{* *}$ & $0,42^{* *}$ & $0,45^{* *}$ \\
SV & - & $0,96^{* *}$ & $-0,55^{* *}$ & $-0,29^{* *}$ & $-0,33^{* *}$ \\
IBES & - & - & $-0,61^{* *}$ & $-0,32^{* *}$ & $-0,36^{* *}$ \\
Depressão & - & - & - & $0,55^{* *}$ & $0,60^{* *}$ \\
Ansiedade & - & - & - & - & $0,62^{* *}$ \\
\hline
\end{tabular}

Nota. Violência Direta (VD); Violência Indireta (VI); Escala de Depressão; Ansiedade e Estresse (EDAE-A); Afetos Positivos; Afetos Negativos (AN); Satisfação de Vida (SV); Índice de Bem-Estar Subjetivo (IBES). ${ }^{*} p<0,05 ;{ }^{* *} p<0,001$

Com o objetivo de verificar quais as variáveis estavam indiretamente associadas à ocorrência de sintomas de ansiedade, depressão e estresse a partir da escala EDAE-A, foi realizada uma análise de Regressão Linear Múltipla pelo método Stepwise (Tabela 3). As variáveis independentes foram o sexo, exposição à
Violência Direta (VD) e Violência Indireta (VI), Afetos Positivos (AP), Afetos Negativos (AN), Satisfação de vida (EMSVA) e IBES (Índice de Bem-Estar Subjetivo). Cabe ressaltar que a variável sexo foi transformada e entrou como variável preditora binária (0 sexo feminino e 1 sexo masculino).

Tabela 3

Modelo de Regressão Linear Múltipla para a EDAE-A (Método Stepwise)

\begin{tabular}{lcccc}
\hline \multicolumn{2}{c}{ Variáveis preditoras incluídas na equação } & \multicolumn{3}{c}{ EDAE-A } \\
\cline { 2 - 5 } & $B$ & Beta & $t$ & \multicolumn{1}{c}{$t$} \\
\hline Afetos Negativos & 0,41 & 0,39 & 8,83 & $<, 001$ \\
Violência Direta & 0,89 & 0,15 & 3,31 & 0,001 \\
Violência Indireta & 0,19 & 0,15 & 3,29 & 0,001 \\
Sexo masculino & $-3,66$ & $-0,17$ & $-4,61$ & $<0,001$ \\
Satisfação de Vida & $-0,07$ & $-0,18$ & $-4,33$ & $<0,001$ \\
Constant & 14,07 & - & 3,57 & $<0,001$ \\
\hline
\end{tabular}

O modelo que melhor explicou essa relação incluiu a associação positiva com as escalas AN, VD e VI e negativa com sexo masculino e SV, sendo este modelo significativo $(\mathrm{F}(5,419)=74,08 ; p<0,001)$. As variáveis incluídas no modelo explicaram $47 \%$ da variação nos escores da EDAE-A.

Outra análise de regressão linear múltipla foi realizada com o objetivo de investigar as variáveis independentemente associadas ao bem-estar subjetivo (BES) dos adolescentes a partir do Índice de Bem-Estar Subjetivo (IBES) (afetos positivos, negativos e satisfação de vida), considerando-se sexo, VD, VI, EDAE-A e subescalas ansiedade, depressão e estresse (Tabela 4).

Os resultados indicam que apenas a subescala depressão e a exposição à VD associam-se ao IBES, sendo a associação negativa. O modelo é estatisticamente significativo $(\mathrm{F}(2,422)=135,08 ; p<0,001)$ e capaz de explicar $39 \%$ na variação nos escores do IBES.

Tabela 4

Modelo de Regressão Linear Múltipla para Índice de Bem-Estar Subjetivo (IBES) (Método Stepwise)

\begin{tabular}{|c|c|c|c|c|}
\hline \multirow{2}{*}{ Variáveis preditoras incluídas no modelo } & \multicolumn{4}{|c|}{ IBES } \\
\hline & $B$ & Beta & $t$ & $p$ \\
\hline Subescala Depressão & $-5,76$ & $-0,60$ & $-15,17$ & $<0,001$ \\
\hline Violência Direta & $-2,16$ & $-0,89$ & $-2,27$ & 0,024 \\
\hline Constant & 253,91 & - & 100,01 & $<0,001$ \\
\hline
\end{tabular}




\section{Discussão}

Este estudo teve como objetivo verificar relações entre exposição à violência direta (ser vítima) e indireta (testemunhar ou ouvir falar sobre), bem-estar subjetivo e sintomas de depressão, ansiedade, estresse em adolescentes escolares de 12 a 18 anos. Foi possível verificar que as variáveis AN, VD e VI, além da associação negativa com sexo masculino e SV, são capazes de explicar $47 \%$ na variação dos escores da escala EDAE-A. Esse resultado demonstra um conjunto importante de variáveis que devem ser consideradas no atendimento de adolescentes que apresentam sintomas internalizantes. Pesquisas têm indicado que ser vítima de violência (VD) possui impacto significativo na vida dos adolescentes, embora nem sempre exista associação com sintomas de depressão, ansiedade e estresse. Neste estudo, os resultados encontrados são consistentes com outras pesquisas que verificaram que a exposição à violência direta esteve relacionada a sintomas de ansiedade e depressão (Hildebrand, Celeri, Morcillo, \& Zanolli, 2015), Transtorno do Estresse Pós-Traumático (Gava, Silva, \& Dell'Aglio, 2013), além de suicídio (Braga \& Dell'Aglio, 2013). No entanto, os resultados ainda sugerem que a exposição a VI também é fator de risco para sintomas de internalização em adolescentes, o que deve ser melhor investigado em trabalhos nacionais.

Apesar de escassos, os estudos sobre exposição à VI confirmam que presenciar, testemunhar ou ouvir falar de violência também apresentam impacto ao desenvolvimento psicológico de adolescentes (Benetti et al., 2014; Benetti, Pizetta, Schwartz, Hass, \& Melo, 2010). No entanto, há divergências entre estudos, sendo que alguns indicam a associação entre exposição a VI e sintomas internalizantes, principalmente em relação ao sexo feminino (Benetti et al., 2010; Zinzow et al., 2009), alguns estudos encontram baixos níveis de sintomas internalizantes associados à exposição VI (Zinzow et al., 2009), e outros não encontram tal associação (Fowler et al., 2009; Kirk \& Hardy, 2014). Pesquisa nacional que utilizou o mesmo instrumento para verificar exposição à violência indicou que ambas as formas de exposições (VD e VI) estiveram associadas a sintomas externalizantes e internalizantes apenas nas meninas (Benetti et al., 2010). Neste estudo, também foi observada associação entre exposição à violência e sintomas de internalização apenas para o sexo feminino, o que sugere que as meninas são mais reativas à exposição, corroborando estudos internacionais (Barry et al., 2015; Bartels, Cacioppo, van Beijsterveldt, \& Boomsma, 2013; Franzese, Covey, Tucker, McCoy, \& Menard, 2014). A inconsistência nos achados relativos à exposição à VI e sintomas de internalização pode ser devido à diversidade de instrumentos utilizados para medir tanto a exposição a esse tipo de violência quanto aos sintomas internalizantes. Embora nem sempre com resultados semelhantes, a maioria dos estudos nacionais e internacionais afirma que, de fato, sintomas de internalização são mais comuns em meninas do que em meninos (Caires, \& Silva, 2011; Azevedo, \& Matos, 2014). Pode-se compreender tal distinção em uma perspectiva de gênero, relacionada a diferenças na socialização entre meninos e meninas, pelas modificações biológicas do período vital da puberdade ou ainda, pela diferença genética relacionada aos sexos (APA, 2014). Além disso, tais diferenças podem ser observadas na forma como são expressos sentimentos e emoções, sendo que, geralmente, meninas são encorajadas a demonstrar seus sentimentos e os meninos a ser mais agressivos (Saffioti, 2008).

A associação positiva entre afetos negativos (AN) e sintomas de ansiedade, depressão e estresse pode ser decorrente da semelhança nos sintomas dessas variáveis verificados nos instrumentos utilizados para avaliar tais construtos. Por exemplo, dentre os afetos negativos, encontram-se "culpado", “deprimido", “desanimado", "irritado" (Segabinazi et al., 2012), características muitas vezes encontradas em adolescentes com diagnóstico de depressão (Argimon, Terroso, Barbosa, \& Lopes, 2013), e presentes no instrumento EDAE-A ("Eu não conseguia ter sentimentos positivos", "Em geral, tive reações exageradas às situações", "Notei que estava ficando agitado"). Dessa forma, observa-se similaridade entre as palavras do instrumento que avalia os afetos negativos e características dos sintomas de depressão, ansiedade e estresse.

Sobre as variáveis associadas ao bem-estar subjetivo, apenas a subescala depressão e violência direta apresentaram associação significativa, sendo ambas negativas, e explicaram 39\% da variação do IBES. Esse resultado enfatiza a importância de se considerar os sintomas depressivos e a exposição a situações de violência, no trabalho preventivo em busca de maior bem-estar entre adolescentes. Alguns estudos têm revelado o quanto ser exposto a eventos estressores e violência afeta de maneira negativa o desenvolvimento (Coyle \& Vera, 2013). Neste estudo, o IBES (que inclui a avaliação da SV juntamente com AP e AN) foi associado negativamente apenas à subescala depressão e não a outros sintomas internalizantes (ex.: ansiedade e estresse), diferentemente de outros estudos, principalmente internacionais, que encontraram tal associação (Derdikman-Eiron et al., 2011). A associação entre depressão e bem-estar subjetivo foi investigada no estudo realizado por Baptista, Hauck Filho e Cardoso (2016), com o objetivo de explicar essa relação a partir do teste de modelos confirmatórios. Os pesquisadores referem que o melhor modelo para explicar essa associação é o bifator o qual indica que depressão e bem-estar subjetivo compartilham um contínuo, mas possuem componentes específicos, sendo um fator geral interpretado como uma dimensão ampla de psicopatologia-saúde que engloba aspetos centrais de características da depressão e o fator específico que apreende aspectos relacionados aos positivos. Assim, os autores indicam que, a partir desse modelo confirmatório, uma parte da saúde mental é 
independente da doença, isto é, uma pessoa pode ter depressão e mesmo assim ter afetos positivos.

Embora no segundo modelo de regressão deste estudo a ansiedade e estresse não foram capazes de explicar as variações nos escores do IBES, a SV e AN, avaliados individualmente, estão relacionados com os sintomas - como indicado na primeira regressão, tendo a escala EDAE-A como variável dependente. Esse aspecto permite comparar tais resultados com outros estudos, separadamente, considerando cada variável, ou em conjunto, por meio do BES. Por exemplo, a associação negativa encontrada entre satisfação de vida (SV) e sintomas de depressão e ansiedade foi também verificada em outro estudo (Gilman \& Huebner, 2006) o qual demonstrou que indivíduos satisfeitos com suas vidas são menos prováveis de apresentar sintomas de psicopatologia diante de eventos estressores. Outro estudo (Proctor, Linley, \& Maltby, 2010) indicou que adolescentes que reportam altos níveis de satisfação com a vida apresentam menos sintomas depressivos, menos afetos negativos e estresse quando comparados com adolescentes com baixos escores na satisfação com a vida. Esses resultados indicam que essas variáveis podem se retroalimentar, considerando que o indivíduo com satisfação de vida tende a apresentar menos sintomas, e, ao contrário, a pessoa com menos sintomatologia tende a perceber sua vida de forma mais positiva (Bartels et al., 2013).

Estudo quantitativo, realizado com 8.984 adolescentes noruegueses de 13 a 19 anos, indicou associação entre sintomas de ansiedade e depressão e baixos níveis de bem-estar subjetivo, especialmente entre meninos (Derdikman-Eiron et al., 2011). Outro estudo, realizado com adolescentes holandeses de 12 a 20 anos, indicou associação negativa entre bem-estar subjetivo e sintomas de depressão e ansiedade (Bartels et al., 2013). É possível compreender que adolescentes com mais sintomas de depressão apresentam menores níveis de bem-estar subjetivo pelo fato de possuírem uma visão pessimista sobre si, o mundo e o futuro (Powell, Abreu, Oliveira, \& Sudak, 2008), já que o BES engloba avaliação cognitiva e afetiva. $\mathrm{O}$ fato de a ansiedade e o estresse não se relacionarem ao BES no modelo da regressão, neste estudo, pode indicar que apesar dos sintomas estarem presentes, os adolescentes avaliam sua vida como boa. Esse aspecto pode sugerir que, assim como na depressão (Baptista et al., 2016), na ansiedade e no estresse também pode haver um fator específico que explicaria esse aspecto. No entanto, a direção dessa relação e o modelo teórico confirmatório da relação entre ansiedade, estresse e bem-estar subjetivo em adolescentes deve ser melhor investigado.

Em conjunto, os resultados sugerem que há controvérsias nessas associações e que pode haver fatores que mediam ou moderam as relações entre exposição à violência, sintomas internalizantes e bem-estar subjetivo. Por exemplo, embora a VI tenha demonstrado associação com sintomas internalizantes, não se associou ao BES, podendo indicar que, mesmo com sintomas de depressão, ansiedade e estresse, os adolescentes expostos de maneira indireta à violência podem ter bons níveis de bem-estar subjetivo ou que os bons níveis de bem-estar subjetivo dos adolescentes expostos à violência indireta os protege dos sintomas de internalização. Além disso, neste estudo, não foi observada associação entre BES, idade e sexo, diferentemente de estudos que indicam tais relações (Poletto \& Koller, 2011; Segabinazi et al., 2012; Segabinazi et al., 2010).

Os resultados das análises realizadas revelaram que tanto a exposição a VD quanto VI e maiores níveis de afetos negativos foram positivamente associados com sintomas de ansiedade, depressão e estresse. Além disso, ser do sexo feminino e ter menores níveis de satisfação de vida, também se mostraram relacionados aos sintomas internalizantes. Esses resultados indicam a importância da proteção contra à exposição à violência, tanto direta quanto indireta, durante a infância e adolescência, tendo em vista seu impacto no desenvolvimento emocional.

Aspecto importante a ser ressaltado é que os estudos utilizam diferentes instrumentos para avaliar exposição à violência e sintomas internalizantes, dificultando comparações. Neste estudo, por limitações do instrumento utilizado, não foi possível verificar características importantes da exposição à violência, tais como os contextos em que ocorrem (se intra ou extrafamiliar), tipos de violência (negligência, física, psicológica ou sexual), tempo, frequência de exposição e proximidade afetiva com a vítima da violência presenciada (no caso da exposição indireta). Dessa forma, destaca-se a importância do desenvolvimento de instrumentos que investiguem mais detalhadamente as características dos diferentes contextos, tipos e formas de violência - principalmente relacionadas à exposição à violência indireta, menos estudada em contexto nacional, a fim de verificar outras associações.

O mesmo deve ser destacado quanto à avaliação do BES, a qual foi realizada por meio do compósito que resultou o IBES, unindo instrumento de dimensão cognitiva (SV) e afetiva (AP e AN). Embora, separadamente, tenha sido observada associação entre SV, AN e sintomas internalizantes, o IBES não se associou a eles. Da mesma forma, apenas a depressão foi associada ao IBES. Esses aspectos devem ser melhor investigados, já que a maior parte dos estudos avalia, separadamente, tais construtos (Poletto \& Koller, 2011; Coyle \& Vera, 2013) e os estudos que os avaliam em conjunto utilizam-se de outros instrumentos (Bartels et al., 2013) o que compromete comparações. Cabe ainda destacar que a idade e o sexo dos adolescentes não foram associados ao IBES, diferentemente de resultados de pesquisas anteriores.

Para estudos futuros, sugere-se a inserção de outras variáveis que influenciam o bem-estar subjetivo, bem como a avaliação de mediadores e moderadores da exposição à violência, utilizando-se um modelo de equações estruturais. Além disso, estudos futuros 
podem avaliar as propriedades psicométricas de tal índice, além de utilizá-lo em outras populações de adolescentes (clínicos e não clínicos, por exemplo). Estudos longitudinais e qualitativos também podem ajudar na compreensão de tais diferenças.

De fato, ainda há incertezas sobre quais fatores ou variáveis influenciam e são preditores mais significativos no BES e mediam ou moderam relações com outros construtos. Considerando a complexidade do construto, pode-se compreender que uma abordagem interacionista que abarque características pessoais, eventos de vida e variáveis contextuais é a melhor forma para compreensão do BES. Além disso, estudos longitudinais podem ajudar na compreensão de como a violência, tanto direta como indireta, pode impactar a qualidade de vida dos adolescentes ao longo do tempo.

\section{Referências}

American Psychiatric Association. (2014). Manual diagnóstico e estatístico de transtornos mentais: DSM-5 (5 ed.). Porto Alegre: Artmed.

Argimon, I. I. L, Terroso, L. B., Barbosa, A. S., \& Lopes, R. M. F. (2013). Intensidade de sintomas depressivos em adolescentes através da escala de depressão de Beck (BDI-II). Boletim Academia Paulista de Psicologia, 33(85), 354-372.

Azevedo, A., \& Matos, A. (2014). Ideação suicida e sintomatologia depressiva em adolescentes. Psicologia, Saúde e Doenças, 15(1), 180-191. doi: $10.15309 / 14 p s d 150115$

Baptista, M., Hauck Filho, N., \& Cardoso, C. (2016). Depressão e bem-estar subjetivo em crianças e adolescentes: Teste de modelos teóricos. Psico, 47(4), 259-267. doi: 10.15448/1980-8623.2016.4.23012

Barbetta, P. A. (2001). Estatística aplicada às ciências sociais. Florianópolis: UFSC.

Bartels, M., Cacioppo, J. T., van Beijsterveldt, T. C., \& Boomsma, D. I. (2013). Exploring the association between well-being and psychopathology in adolescents. Behaviour Genetic, 43(3), 177-190. doi: 10.1007/s10519-013-9589-7

Barry, S. A., Rabkin, A. N., Olezeski, C. L., Rivers, A. J., \& Gordis, E. B. (2015). Relation between aggression exposure in adolescence and adult posttraumatic stress symptoms: Moderating role of the parasympathetic nervous system. Physiology Behavior, 15, 97-102. doi: 10.1016/j.physbeh.2015.01.006

Benetti, S. P. C., Pizetta, A., Schwartz, C. B., Hass, R. A., \& Melo, V. L. (2010). Problemas de saúde mental na adolescência: características familiares, eventos traumáticos e violência. Psico-USF, 15(3), 321-332.

Benetti, S., P., C., Schwartz, C., Soares, G. R., Macarena, F., Patussi, M. M. (2014). Psychosocial adolescent psychosocial adjustment in Brazil - perception of parenting style, stressful events and violence. International Journal of Psychological Research, 7(1), 40-48.

Braga, L. L., \& Dell'Aglio, D. D. (2013). Suicídio na adolescência: Fatores de risco, depressão e gênero. Contextos Clínicos, 6(1), 2-14. doi: 10.4013/ctc.2013.61.01

Brasil, Conselho Nacional de Saúde. (2013). Diretrizes e normas para pesquisa envolvendo seres humanos. RESOLUÇÃO No 466, de 12 de dezembro de 2012. Brasília: Diário Oficial da União; Poder Executivo.

Caires, S., \& Silva, C. (2011). Fatores de stress e estratégias de coping entre adolescentes no $12^{\circ}$ ano de escolaridade. Estudos de Psicologia, 28(3), 295-306. doi: 10.1590/S0103-166X2011000300001

Camargo, S. P. H., Abaid, J. L. W., \& Giacomoni, C. H. (2011). Do que eles precisam para serem felizes? A felicidade na visão de adolescentes. Psicologia Escolar e Educacional, 15(2), 241-250.

Carey, D. C., \& Richards, M. H. (2014). Exposure to community and social maladjustment among urban African American Youth. Journal of Adolescence, 37(7), 1161-1170. doi: 10.1016/j.adolescence.2014.07.021

Carlos, D. M., Ferriani, M. G. C., Esteves, M. R., Silva, L. M. P., \& Scatena, L. (2014). O apoio social sob a ótica de adolescentes vítimas de violência doméstica. Revista da Escola de Enfermagem da USP, 48(4), 610-617. doi: 0.1590/S0080-623420140000400006

Coyle, L. D., \& Vera, E. M. (2013). Uncontrollable stress, coping, and subjective well-being in urban adolescents. Journal of Youth Studies, 16(3), 391-403. doi: 0.1080/13676261.2012.756975

Derdikman-Eiron, R., Indredavik, M. S., Bratberg, G. H., Taraldsen, G., Bakken, I. J., \& Colton, M. (2011). Gender differences in subjective well-being, self-esteem and psychosocial functioning in adolescents with symptoms of anxiety and depression: Findings from the Nord-Trøndelag health study. Scandinavian Journal of Psychology, 52(3), 261-267. doi: 10.1111/j.1467-9450.2010.00859.x

Diener, E. (2000). Subjective well-being: The science of happiness and a proposal for a national index. American Psychologist, 55(1), $34-43$.

Fowler, P. J., Tompsett, C. J., Braciszewski, J. M., Jacques-Tiura, A. J., \& Baltes, B. B. (2009). Community violence: A meta-analysis on the effect of exposure and mental health outcomes of children and adolescents. Development Psychopathology, 21(1), 227-59. doi: 10.1017/S0954579409000145

Franco, G. R., \& Rodrigues, M. C. (2014). Programas de intervenção na adolescência: Considerações sobre o desenvolvimento positivo do jovem. Temas em Psicologia, 22(4), 677-690.

Franzese, R. J., Covey, H. C., Tucker, A. C., McCoy, L., \& Menard, S. (2014). Adolescent exposure to violence and adult physical and mental health problems. Child Abuse \& Neglect, 38(12), 1955-1965. doi: 10.1016/j.chiabu.2014.10.017

Gava, L. L., Silva, D. G., \& Dell'Aglio, D. D. (2013). Sintomas e quadros psicopatológicos identificados nas perícias em situações de abuso sexual infanto-juvenil. Psico, 44(2), 235-244.

Gilman, R., \& Huebner, E. S. (2006). Characteristics of adolescents who report very high lifes satisfaction. Journal of Youth and Adolescence, 35(3), 293-301. doi:0.1007/s10964-006-9036-7

Hildebrand, N. A., Celeri, E. H. R. V., Morcillo, A. M., \& Zanolli, M. L. (2015). Violência doméstica e risco para problemas de saúde mental em crianças e adolescentes. Psicologia: Reflexão e Crítica, 28(2), 213-221. doi: 10.1590/1678-7153.201528201

Kirk, D. S., \& Hardy, M. (2014). The acute and enduring consequences of exposure to violence on youth mental health and aggression. Justice Quarterly, 31(3), 539-567. doi: 10.1080/07418825.2012.737471 
Long, R. F., Huebner, S. E, Wedell, D. H., \& Hills, K. J. (2012). Measuring school-related subjective well-being in adolescents. American Journal of Orthopsychiatry, 82(1), 50-60. doi: 10.1111/j.1939-0025.2011.01130.x

Maranhão, J. H., Coêlho, J. P. L., Lopes, G. S., Colaço, V. F. R., \& Santos, W. S. (2014). Violência, risco e proteção em estudantes de escola pública. Fractal, 26(2), 429-444.

Margolin, G., Vickerman, K. A., Oliver, P. H., \& Gordis, E. B. (2010). Violence exposure in multiple interpersonal domains: Cumulative and differential effects. Journal of Adolescent Health, 47(2), 198-205. doi: 10.1016/j.jadohealth.2010.01.020

Margolin, G., \& Gordis E. B. (2000). The effects of family and community violence on children. Annual Review Psychological, 51, 445-479. doi: 10.1146/annurev.psych.51.1.445

Mendonça, H., Ferreira, M. C., Caetano, A., \& Torres, C. V. (2014). Cultura organizacional, coping e bem-estar subjetivo: um estudo com professores de universidades brasileiras. Revista Psicologia Organizações e Trabalho, 14(2), 230-244.

Mrug, S., \& Windle, M. (2010). Prospective effects of violence exposure across multiple contexts on early adolescents' internalizing and externalizing problems. Journal of Child Psychology and Psychiatry, 51 (8), 953-961. doi: 10.1111/j.1469-7610.2010.02222

Oliveira, S. F., Queiroz, M. I. N., \& Costa, M. L. A. (2012). Bem-estar subjetivo na terceira idade. Motricidade, 8(2), 1038-1047.

Park, N. (2004). The role of subjective well-being in positive youth development. The Annals of the American Academy of Political and Social Science, 591(1), 25-39.

Patias, N. D., Machado, W. L., Bandeira, D. R., \& Dell'Aglio, D. D. (2016). Depression anxiety and stress scale (DASS-21) - Short Form: Adaptação e validação para adolescentes brasileiros. Psico-USF, 21(3), 459-469. doi: 10.1590/1413-82712016210302

Poletto, M., \& Koller, S. H. (2011). Subjective well-being in socially vulnerable children and adolescents. Psicologia: Reflexão e Crítica, 24(3), 476-484. doi: 10.1590/S0102-79722011000300008

Powell, V. B., Abreu, N., Oliveira, I. R., \& Sudak, D. (2008). Terapia cognitivo-comportamental da depressão. Revista Brasileira de Psiquiatria, 30(2), 73-80.

Pratta, E. M. M., \& Santos, M. A. (2007). Família e adolescência: A influência do contexto familiar no desenvolvimento psicológico dos seus membros. Psicologia em Estudo, 12(2), 247-256. doi.org/10.1177/0272431603260879

Proctor, C., Linley, P. A., \& Maltby, J. (2010). Very happy youths: Benefits of very high life satisfaction among adolescents. Social Indicators Research, 98(3), 519-532. doi: 0.1007/s11205-009-9562-2

Richters, J. E., \& Martinez, P. (1993). The NIMH community violence project: I. Children as victims of and witnesses to violence. Psychiatry, $56(1), 7-21$.

Saffioti, H. (2008). Gênero: Ontogênese e filogênese. In S. L. Kato (Ed.), Manual de capacitação multidisciplinar (Lei n. 11.340 , de 07 de agosto de 2006 - Lei Maria da Penha). Tribunal de Justiça do Mato Grosso. Cuiabá: Departamento Gráfico-TJMT, pp. 91-124.

Scorsolini-Comin, F., \& Santos, M. A. (2011). Relações entre bem-estar subjetivo e satisfação conjugal na abordagem da psicologia positiva. Psicologia: Reflexão e Crítica, 24(2), 658-665. doi: 10.1590/S0102-79722011000400005

Segabinazi, J. D., Giacomoni, C. H., Dias, A. C. G., Teixeira, M. A. P., \& Moraes, D. A. O. (2010). Desenvolvimento e validação preliminar de uma escala multidimensional de satisfação de vida para adolescentes. Psicologia: Teoria e Pesquisa, 26(4), 653-659. doi: 10.1590/S010237722010000400009

Segabinazi, J. D., Zortea, M., Zanon, C., Bandeira, D. R., Giacomoni, C. H., \& Hutz, C. S. (2012). Escala de afeto positivo e negativo para adolescentes: Adaptação, normatização e evidências de validade. Avaliação Psicológica, 11(1), 1-12.

Seligman, M. E., \& Csikszentmihalyi, M. (2000). Positive psychology: An introduction. American Psychologist, 55(1), 5-14.

Steinberg, L., \& Lerner, R. M. (2004). The scientific study of adolescence: A brief history. Journal of Early Adolescence, $24,45-54$. doi:0.1177/0272431603260879

Zavaschi, M. L., Benetti, S., Polanczyk, G. V., Solés, N., \& Sanchotene, M. L. (2002). Adolescents exposed to physical violence in the community: A survey in Brazilian public schools. Revista Panamericana de Salud Publica, 12(5), 327-332.

Zinzow, H. M., Ruggiero, K. J., Resnick, H., Hanson, R., Smith, D., Saunders, B., \& Kilpatrick, D. (2009). Prevalence and mental health correlates of witnessed parental and community violence in a national sample of adolescents. Journal of Child Psychology and Psychiatry, 50(4), 441-450. doi:10.1111/j.1469-7610.2008.02004.x

Waiselfisz, J. J. (2014). Juventude VIVA - Mapa da violência 2014: Homicídios e juventude no Brasil. Brasília: Qualidade.

Woyciekoski, C., Natividade, J. C., \& Hutz, C. S. (2014). As contribuições da personalidade e dos eventos de vida para o bem-estar subjetivo. Psicologia: Teoria e Pesquisa, 30(4), 401-409.

\section{Sobre as autoras}

Naiana DapievePatias é Psicóloga, Mestre (UFSM) e Doutora em Psicologia (UFRGS), docente nos cursos de Graduação e Mestrado em Psicologia na Faculdade Meridional, IMED, Passo Fundo, RS.

Júlia Assumpção Heine é Psicóloga formada pela UFRGS e aluna do curso de especialização em Teoria Psicanalítica e as Psicoterapias na Infância e Adolescência, no Contemporâneo:Instituto de Psicanálise e Transdisciplinaridade.

Débora Dalbosco Dell'Aglio é Psicóloga, Mestre e Doutora em Psicologia do Desenvolvimento (UFRGS), Professora Pesquisadora PPG em Educação UniLassale/Canoas e Professora Colaboradora PPG Psicologia UFRGS, RS. 